\title{
Estructura interna y confiabilidad del BDI (Beck Depression Inventory) en universitarios de Michoacán (México)
}

\author{
Ferran Padrós ${ }^{1}$ y Blanca Edith Pintor Sánchez ${ }^{2}$
}

\begin{abstract}
RESUMEN
Los trastornos depresivos generan importante discapacidad y son de los más prevalentes en la actualidad (Organización Mundial de la Salud, 2015). Por ello, es necesario disponer de instrumentos psicológicos para detectar dichos trastornos. Una de las escalas más utilizada es el BDI (Beck Depression Inventory), y su primera versión es de uso gratuito. En este trabajo se estudió la estructura y la consistencia internas de la escala BDI (Beck, Rush, Shaw y Emery, 1979) en población universitaria de Michoacán (México). Se aplicó la escala BDI a 1190 universitarios con edades comprendidas entre 17 y 38 años $(M=$ $18.78, D E=1.72)$ y la mayoría fueron mujeres $(n=801,67.3 \%)$. Se aplicó un análisis de Componentes Principales, cuyos resultados sugieren una estructura de dos factores, corroborado por un Análisis Factorial Confirmatorio $\left(\chi^{2} / g l=1.85 ; N F I=.910 ; C F I=.956\right.$; RMSEA =.027). Se observó una elevada consistencia interna de la escala total $(\mathrm{a}=.898) \mathrm{y}$ también resultó adecuada para los 2 factores $(a=.866, \alpha=.701)$. Los resultados indican que el BDI es adecuado para aplicarse a población universitaria en Michoacán. Asimismo, se ofrecen datos descriptivos en población universitaria de Michoacán (México). No se observó relación entre la puntuación total de la escala y la edad, pero sí que la media de las mujeres fue superior a la mostrada por los varones. Destaca que hasta un $13.3 \%$ de la muestra puede cumplir criterios de depresión.
\end{abstract}

Palabras clave: depresión, estructura interna, escala, consistencia interna, estudiantes universitarios.

\section{Internal structure and reliability of the BDI (Beck Depression Inventory) in college students from Michoacán (Mexico)}

\begin{abstract}
Depressive disorders generate significant disability and are among the most prevalent at present (Organización Mundial de la Salud, 2015). Therefore, it is necessary to have psychological instruments to detect these disorders. One of the most used scales is the BDI (Beck Depression Inventory), in its first version is free. In this work we studied the internal structure and reliability of the BDI scale (Beck, Rush, Shaw and Emery, 1979) in university population of Michoacan (Mexico). It was applied the BDI scale to 1190 university students aged between 17 and 38 years $(M=18.78, S D=1.72)$ and the majority were women $(n=801,67.3 \%)$. A Principal component analysis was applied, our results suggest a structure of two factors, corroborated by a Confirmatory Factor Analysis $\left(\chi^{2} / d f=1.85 ; N F I=.910 ; C F I=.956 ; R M S E A=.027\right)$. A high reliability of the total scale was observed $(a=.898)$ and it was also adequate for the 2 factors $(a=.866, a=.701)$. The results indicate that the BDI is adequate to apply to the university population in Michoacan. Descriptive data are also offered in the university population of Michoacan
\end{abstract}

\footnotetext{
${ }^{1}$ Universidad Michoacana de San Nicolás de Hidalgo, México; fpadros@umich.mx; https://orcid.org/00000001-8911-8096

2 Universidad Michoacana de San Nicolás de Hidalgo, México; blanca.pintor@umich.mx; https://orcid.org/0000-0002-1878-7812
} 
(Mexico). No relationship was observed between the total score of the scale and age, but the mean of the women was higher than that shown by the men. Stresses that up to $13.3 \%$ of the sample can meet criteria of depression.

Keywords: depression, internal structure, scale, internal consistency, college students.

Se ha estimado que 350 millones de personas padecen un trastorno depresivo. La depresión se asocia a un gran nivel de discapacidad en el área laboral, social, familiar y dias perdidos por la incapacidad, incluso incide en niveles superiores a los ocasionados por enfermedades crónico-degenerativas (Lara-Muñoz, Medina-Mora, Borges, \& Zambrano, 2007; Organización Mundial de la Salud, 2015). En un reciente metanálisis, se calculó la prevalencia de la depresión general, incidencia (de un año) y de por vida, la cual resultó de $12.9 \%, 7.2 \%$ y $10.8 \%$, respectivamente. Debe destacarse la prevalencia puntual de depresión que resultó significativamente mayor en mujeres (14.4\%) a la hallada en varones (11.5\%) (Lim et al., 2018). En México, la Encuesta Nacional de Evaluación del Desempeño (ENED) 2002-2003, realizada por Bello, Puentes-Rosas, Medina-Mora y Lozano (2005) reportó una prevalencia de depresión del $4.5 \%$ en población general, $5.8 \%$ en mujeres y $2.5 \%$ en hombres. Debe señalarse que algunos autores han observado que las diferencias por género respecto a la presencia de sintomatología depresiva se inician en la adolescencia (Nolen-Hoeksema \& Girgus, 1994; Wade, Cairney, \& Pevalin, 2002). En estudiantes universitarios se ha estimado que un $29,9 \%$ padece depresión leve, 14,2\% moderada y 3,2\% depresión severa (Montoya et al., 2010), superiores a lo descrito por Sanz y Vázquez (1998) en cuyo estudio hallaron que el $13.8 \%$ con posible depresión leve, $4.7 \%$ moderada y solo el $0.4 \%$ grave.

La Organización Mundial de la Salud (OMS) estimó que la depresión será una de las tres principales causas de discapacidad a nivel mundial en el año 2030 (Mathers \& Loncar, 2006). Además, frecuentemente se asocia a otros trastornos mentales como los de ansiedad, adicciones entre otros (Newby, McKinnon, Kuyken, Gilbody, \& Dalgleish, 2015) y presenta una destacada comorbilidad con enfermedades físicas (Kang et al., 2015).

Por ello, es necesario el uso de instrumentos específicos para evaluar la presencia de sintomatología depresiva. En el ámbito hospitalario se utiliza con frecuencia la escala heteroaplicada de Hamilton (Hamilton Depression Rating Sacle; HDRS, Hamilton, 1960), y diversas escalas autoaplicadas como: a) escala de depresión del Centro de Estudios epidemiológicos (CES-D; Radloff, 1977), b) escala de Zung (Zung, 1965) y c) la escala de Montgomery y Asberg (1979). Pero probablemente el instrumento autoadministrado más utilizado sea el BDI (Beck depression inventory) que tiene 21 items, tanto la primera versión (Beck, Ward, Mendelson, Mock, \& Erbaugh, 1961) como la segunda (Beck, Rush, Shaw, \& Emery, 1979) del mismo BDI-I. Según Sanz (2013), el BDI (incluyendo las diferentes versiones) es el instrumento más referenciado cuando se investiga la depresión. Es importante señalar que el BDI se ha basado en la clasificación del DSM y se ha modificado conforme a los cambios de criterios que el diagnóstico del trastorno depresivo mayor ha tenido (Estrada-Aranda, Delgado-Álvarez, Landero-Hernández, \& González-Ramírez, 2015). 
En 1996 se publicó la versión original del BDI-II (Beck, Steer, \& Brown, 1996) adaptada posteriormente en España (Beck, Steer, \& Brown, 2011). Debe señalarse que las propiedades psicométricas del BDI-II son superiores a los del BDI-I y BDI-IA (Beck et al., 2011; Botella \& Ponte, 2011; Sanz \& García-Vera, 2009). Además de que el BDI-II presenta mejoras a nivel de contenido, la más notable entre ellas es que el evaluado informa sobre la presencia de sintomatología depresiva en las últimas semanas- Este es el criterio utilizado en la cuarta edición del Manual Diagnóstico y Estadístico de los Trastornos mentales (DSM-IV, American Psychiatric Association, 1995) con el que se fundamentó la última versión DSM-5 (American Psychiatric Association, 2013), mientras que la versión del BDI-I de 1979 se refiere a la última semana. En el BDI-II se modificaron los ítems que aludian a la pérdida de apetito e insomnio, incluyendo la opción de presencia de aumento de ingesta y de hipersomnia (características de la especificación de sintomas atípicos). Por otro lado, también incluye un ítem que evalúa la presencia de alteraciones de tipo psicomotor (como agitación o retraso psicomotor). Debe destacarse que todos los otros criterios del trastorno depresivo mayor del DSM-5 (American Psychiatric Association, 2013) están contemplados en la versión del BDI-I de 1979. Debe destacarse que el BDI-IA (Beck et al., 1979) y el BDI-II tienen los derechos reservados, puesto que pertenecen a una editorial. En cambio, el BDI-I puede utilizarse de forma gratuita y presenta características psicométricas aceptables, además de ser una escala breve y de fácil corrección e interpretación, lo que hace que sea de gran utilidad.

El BDI en la versión de Beck et al. (1979), el BDI-I, es un instrumento que ha mostrado una estructura interna variable de 2,3 y hasta 4 factores (Sanz, 2013). Asimismo, en los distintos estudios ha presentado adecuada consistencia interna, con valores de alfa de Cronbach de $\mathrm{a}=.78$ a a $=.92$ (Beck, Steer, \& Garbin, 1988). Además, el BDI-I ha mostrado adecuada validez concurrente (Jurado et al., 1998) con otros instrumentos que evalúan la presencia de depresión, como por ejemplo con la HDRS (Hamilton, 1960), la escala de Zung (1965) y la subescala de depresión del MMPI (Hathaway \& McKinley, 1943), ya que se han reportado relaciones con valores entre $r=.72$ y .73. En México, utilizando la escala de Zung, se observó una relación de $r=$ .65 en población general y de $r=.70$ en población clínica (Jurado et al., 1998).

Respecto a la fiabilidad temporal, en España, Sanz y Vázquez (1998) han reportado valores de fiabilidad test-retest de $r=.72$ (en un mes) y de $r=.60$ y $r$ $=.69$ (en tres meses). Finalmente, es destacable que el BDI se ha mostrado como un instrumento con adecuada validez diferencial, mostrando altos niveles de especificidad y sensibilidad al hacer uso de la puntuación 18 (Chan, 1991; Kendall, Hollon, Beck, Hammen, \& Ingram, 1987; Rudd \& Rajab, 1995). Por ello, el objetivo principal de la presente investigación fue estudiar la estructura interna, bondad de los ítems, y la consistencia interna del BDI en jóvenes universitarios de Michoacán. También se pretende ofrecer datos descriptivos sobre los niveles de sintomatología depresiva en dicha población, así como estudiar la posible relación de la puntuación total del BDI con la edad y posibles diferencias en la puntuación del BDI en función del género. 


\section{MÉTODO}

\section{Participantes}

Hasta 1190 estudiantes de diferentes facultades de la Universidad Michoacana de San Nicolás de Hidalgo (de las carreras de Historia, Odontología, Psicología, Contaduría y Ciencias Administrativas) participaron en la presente investigación. El muestreo fue incidental por conveniencia. Los participantes eran jóvenes de entre 17 y 38 años $(M=18.78, D E=1.72)$, y la mayoria, mujeres $(n=801,67.3 \%)$.

\section{Instrumentos}

El BDI-I (Beck Depresion Inventory), escala que se estudia en el presente trabajo, evalúa la presencia de sintomatología depresiva como: tristeza, llanto, pérdida de placer, sentimientos de fracaso y de culpa, pensamientos o deseos de suicidio, pesimismo, entre otros. Contiene 21 items, en cada uno de los cuales se debe escoger entre cuatro afirmaciones (Ausente o minima, Leve, Moderada y Grave; de 0 a 3 puntos) que describen circunstancias donde el evaluado debe indicar aquella con la que mejor se identifique. La escala alude a la posible presencia de sintomatología en la última semana. La puntuación global puede oscilar entre 0 y 63 puntos. Las características psicométricas del BDI se describieron en la introducción. En la presente investigación se utilizó la versión adaptada del BDI-I para población mexicana realizada por Jurado et al. (1998).

\section{Procedimiento}

En primer lugar, el protocolo de investigación fue revisado y aprobado por el comité de ética de la Facultad de Psicología de la Universidad Michoacana de San Nicolás de Hidalgo. Posteriormente, se solicitó permiso en diferentes facultades de la Universidad Michoacana de San Nicolás de Hidalgo, y en aquellas donde se obtuvo permiso, pasantes de psicología administraron el protocolo de recolección de datos a los estudiantes (solo aquellos que aceptaron participar de forma voluntaria). Debe señalarse que los estudiantes no obtuvieron ningún beneficio por responder a los cuestionarios, ni tampoco agravio alguno si se negaban a participar. Previo a responder al cuestionario, firmaron un formato de consentimiento informado donde se les informaba que la participación era voluntaria y anónima. La aplicación fue grupal, se les solicitó la edad y el sexo junto al BDI en una duración aproximada 10 minutos. Se eliminaron 6 participantes que no contestaron la totalidad de la escala.

\section{Análisis de datos}

Se utilizaron los análisis estadísticos siguientes: Análisis de Componentes Principales en el Análisis Factorial Exploratorio con rotación Varimax.Posteriormente se realizó un análisis factorial confirmatorio (AFC) mediante análisis de ecuaciones estructurales y se hizo uso del método de máxima verosimilitud robusto (ML Robusto). También se utilizó el alfa de Cronbach, el alfa ordinal y el índice de atenuación (Domínguez-Lara, 2017), así como el uso de descriptivos como la media aritmética, desviación estándar, frecuencias y porcentajes.

Los análisis se llevaron a cabo con el programa 22.0 (IBM Corp Released, 2013), a excepción del AFC, que utilizó el programa MPlus Versión 7.0 
(Muthén \& Muthén, 2011). El AFC se realizó con los Mínimos Cuadrados Ponderados Robustos y la varianza ajustada (Weighted Least Squares Mean and Variance Adjusted, WLSMV), una aproximación apropiada para el AFC con datos categóricos (Muthén \& Muthén, 2011).

\section{RESULTADOS}

\section{Estructura interna}

La estructura interna del BDI se estudió primero a través de un Análisis de Componentes Principales, debido a la variedad observada en los estudios previos. Primero se corroboró que los datos fueran adecuados para realizar un análisis de componentes principales (Medida de adecuación muestral de Kaiser-Meyer-Olkin =.956; Test de esfericidad de Bartlett $\chi^{2}=7340.709, g l=$ 210, $\mathrm{p}<.001)$. Como puede observarse en la tabla 1, dos componentes obtienen un autovalor superior a la unidad, explicando el $40.21 \%$ de la varianza.

Tabla 1.

Valores propios, varianza explicada y acumulada de los factores del BDI.

\begin{tabular}{cccc}
\hline Componente & Autovalor & $\begin{array}{c}\text { \% Varianza } \\
\text { explicada }\end{array}$ & $\begin{array}{c}\text { \% Varianza explicada } \\
\text { acumulada }\end{array}$ \\
\hline $\mathbf{1}$ & $\mathbf{7 . 2 4}$ & $\mathbf{3 4 . 5 1}$ & $\mathbf{3 4 . 5 1}$ \\
$\mathbf{2}$ & $\mathbf{1 . 1 9}$ & $\mathbf{5 . 6 9}$ & $\mathbf{4 0 . 2 1}$ \\
3 & 0.98 & 4.66 & 44.87 \\
4 & 0.94 & 4.48 & 49.36 \\
5 & 0.84 & 4.02 & 53.38 \\
\hline
\end{tabular}

Posteriormente, se hizo un AFC, donde se consideró la estimación de chicuadrada $\left(\chi^{2}\right)$ y se ajustó a los grados de libertad. En un modelo con un ajuste adecuado, se debe cumplir ausencia de significación y que $\chi^{2} / g l<3$ (Bollen, 1989). También se utilizaron índices de ajuste como: el índice de ajuste comparativo $(C F I)$, el indice de ajuste incremental o indice de ajuste normado $(N F I)$ y el indice de ajuste incremental o índice de ajuste no normado (NNFI), los cuales se consideran que indican un buen modelo cuando muestran valores > .95, (Bentler, 1990; Hu \& Bentler, 1999), y también la raíz media cuadrada del error de aproximación (RMSEA), donde valores de $\leq 0.08$, indican un ajuste razonable (Kline, 1998). Respecto a los resultados, se puede observar que ambos modelos (de un factor y de dos factores relacionados) muestran valores de $\mathrm{x}^{2}$ significativos, y el valor de $N F I$ con valores inferiores a .95, lo cual indica mal ajuste. Además, el modelo de un factor también muestra un valor bajo en el indicador CFI. El modelo de dos factores relacionados muestra un ajuste aceptable, ya que el valor del $\chi^{2} / g l, N N F I, C F I$ y de RMSEA resultaron adecuados, y todos los indices, mejores que los extraídos del modelo unifactorial (véase tabla 2).

Tabla 2.

Índices de ajuste de los modelos de 2 factores relacionados y del modelo de un solo factor.

\begin{tabular}{lccccccc}
\hline Modelo & $\chi^{2}(\boldsymbol{g l})$ & $\chi^{2} / \boldsymbol{g l}$ & $\boldsymbol{p}$ & NFI & NNFI & CFI & RMSEA (90\% IC) \\
\hline 2 factores & $348.25(188)$ & 1.85 & $<.001$ & .910 & .951 & .956 & $.027(.022-.031)$ \\
1 factor & $440.39(189)$ & 2.33 & $<.001$ & .886 & .924 & .931 & $.033(.029-.037)$ \\
\hline
\end{tabular}


Tabla 2.

Índices de ajuste de los modelos de 2 factores relacionados y del modelo de un solo factor.

\begin{tabular}{llllllll}
\hline Modelo & $\chi^{2}(\boldsymbol{g l})$ & $\chi^{2} / g I$ & $\boldsymbol{p}$ & NFI & NNFI & CFI & RMSEA $(\mathbf{9 0 \%}$ IC) \\
\hline Nota: $\chi^{2}=$ Chi-cuadrado Satorra-Bentler; $g l=$ grados de libertad; $p=$ significación estadística; $\chi^{2} / g l ;$ Chi-cuadrado \\
dividido por los grados de libertad; $N F I=$ Normed Fit Index; $N N F I=$ Non Normed Fit Index; $C F I=$ Comparative fit index; \\
RMSEA = Root mean square error of approximation; $I C=$ Intervalo de Confianza.
\end{tabular}

\section{Consistencia interna}

Respecto a la consistencia interna, y siguiendo las recomendaciones de Dominguez-Lara (2017), debido a que las opciones de respuesta son cuatro, se calculó el alfa ordinal $\left(\mathrm{a}_{\mathrm{O}}\right)$, el alfa de Cronbach $\left(\mathrm{a}_{\mathrm{C}}\right)$ y se estimó el índice de Atenuación (IA) de la escala total y de cada uno de los factores. El valor de alfa del total de la escala resultó aceptable $\left(\mathrm{a}_{\mathrm{O}}=.914\right.$, el $\mathrm{a}_{\mathrm{C}}=.898$ e IA del $\left.2 \%\right)$. Respecto a los factores, para el factor creencias y sentimientos resultó también adecuado ( $\mathrm{a}_{\mathrm{O}}=.881$, el $\mathrm{a}_{\mathrm{C}}=.866$ e IA del $2 \%$ ) y aceptable para el de sintomatología vegetativa ( $\mathrm{a}_{\mathrm{O}}=.75$, el $\mathrm{a}_{\mathrm{C}}=.701$ e IA del $6 \%$ ). Teniendo en cuenta los valores del IA, los cuales oscilaron entre el 2 y el 6\%, puede concluirse que el estimador del alfa de Cronbach es un estimador apropiado, ya que porcentajes inferiores al 30\% indican una atenuación no significativa (Dominguez-Lara, 2017).

\section{Descripción de los ítems}

Todas las puntuaciones medias y de la desviación estándar de los ítems fueron inferiores a la unidad. Asimismo, puede observarse que los valores de asimetría y curtosis indican que la distribución de la mayoría de los reactivos no sigue la ley normal (ver tabla 3).

Tabla 3.

Media, desviación estándar, asimetría y curtosis para cada ítem.

\begin{tabular}{ccccc}
\hline Ítems & Media & Desviación típica & Asimetría & Curtosis \\
\hline 1 & 0.51 & 0.62 & 1.03 & 1.01 \\
2 & 0.33 & 0.66 & 2.16 & 4.83 \\
3 & 0.30 & 0.65 & 2.28 & 4.64 \\
4 & 0.55 & 0.79 & 1.59 & 2.24 \\
5 & 0.34 & 0.57 & 1.66 & 2.78 \\
6 & 0.41 & 0.78 & 2.20 & 4.37 \\
7 & 0.40 & 0.71 & 1.68 & 1.95 \\
8 & 0.45 & 0.64 & 1.41 & 2.11 \\
9 & 0.21 & 0.49 & 2.76 & 9.36 \\
10 & 0.43 & 0.82 & 2.07 & 3.52 \\
11 & 0.73 & 0.97 & 1.33 & 0.79 \\
12 & 0.41 & 0.65 & 1.62 & 2.46 \\
13 & 0.65 & 0.78 & 0.87 & -0.26 \\
14 & 0.47 & 0.85 & 1.74 & 1.97 \\
15 & 0.51 & 0.68 & 1.06 & 0.34 \\
16 & 0.56 & 0.69 & 1.14 & 1.21 \\
17 & 0.49 & 0.66 & 1.38 & 2.44 \\
18 & 0.38 & 0.65 & 1.83 & 3.27 \\
19 & 0.29 & 0.58 & 2.24 & 5.23 \\
20 & 0.43 & 0.61 & 1.25 & 1.25 \\
21 & 0.21 & 0.57 & 2.94 & 8.65 \\
\hline
\end{tabular}


Las correlaciones de cada ítem con la puntuación de la escala total corregida (es decir, sin tener en cuenta dicho item) fueron superiores a $.30 \mathrm{y}$ superiores a .40 (excepto los ítems 11 y 19). Destaca que la eliminación del item 11 hace aumentar el valor del alfa de Cronbach de la escala total (ver tabla 4).

\begin{tabular}{|c|c|c|}
\hline Ítems & $\begin{array}{l}\text { Correlación ítem } \\
\text { escala total (corregida) }\end{array}$ & $\begin{array}{l}\text { Alfa de la escala total } \\
\text { si se elimina ítem }\end{array}$ \\
\hline 1 & .57 & .89 \\
\hline 2 & .54 & .89 \\
\hline 3 & .63 & .89 \\
\hline 4 & .57 & .89 \\
\hline 5 & .60 & .89 \\
\hline 6 & .50 & .89 \\
\hline 7 & .64 & .89 \\
\hline 8 & .60 & .89 \\
\hline 9 & .56 & .89 \\
\hline 10 & .50 & .89 \\
\hline 11 & .35 & .90 \\
\hline 12 & .53 & .89 \\
\hline 13 & .53 & .89 \\
\hline 14 & .56 & .89 \\
\hline 15 & .53 & .89 \\
\hline 16 & .52 & .89 \\
\hline 17 & .50 & .89 \\
\hline 18 & .46 & .89 \\
\hline 19 & .32 & .89 \\
\hline 20 & .41 & .89 \\
\hline 21 & .46 & .89 \\
\hline
\end{tabular}

\section{Descriptivos y relación con la edad y género del BDI-I en población de jóvenes} universitarios de Michoacán (México)

La media de la puntuación total de la escala fue de $9.03(\mathrm{DE}=8.34)$, la moda resultó 0 y los valores totales oscilaron de 0 a 55. En la tabla 5, se ofrecen los valores de frecuencias y porcentajes (simples y acumulados) de la puntuación total del BDI según los criterios de gravedad propuestos por Beck y Steer (1993). Destaca que el 13.3\% de los que respondieron obtuvieron una puntuación igual o superior a 18, que indica probable trastorno depresivo. 
Tabla 5.

Frecuencias y porcentajes de los valores del BDI según criterios de gravedad Beck y Steer (1993).

\begin{tabular}{lcccc}
\hline $\begin{array}{l}\text { Gravedad de } \\
\text { depresión }\end{array}$ & $\begin{array}{c}\text { Rango de } \\
\text { Puntuación }\end{array}$ & Frecuencia & $\%$ & $\begin{array}{c}\text { Porcentaje } \\
\text { acumulado }\end{array}$ \\
\hline Ausente o mínima & $0-9$ & 755 & 63.8 & 63.8 \\
Leve & $10-16$ & 250 & 21.11 & 84.9 \\
Moderada & $17-29$ & 141 & 11.91 & 96.8 \\
Grave & $30-63$ & 38 & 3.21 & 100 \\
\hline
\end{tabular}

No se observó correlación entre la edad y la puntuación del BDI $(r=.018$; $p=.547)$, pero la puntuación media del género femenino $(M=9.62 ; D E=8.53)$ resultó significativamente superior $\left(t_{1182}=3.520 ; p<.001\right)$ a la obtenida por los participantes masculinos $(M=7.81 ; D E=7.80)$.

\section{DISCUSIÓN}

En este trabajo se estudió la estructura interna y la confiabilidad del BDI, una escala que evalúa la presencia e intensidad de sintomatología depresiva.

Respecto a la estructura interna, la solución de dos factores relacionados fue más adecuada que la de un solo factor, y explica un porcentaje de varianza por encima del 40\%, aunque en el AFC se observó un ajuste algo dudoso. Debe recordarse que respecto a la estructura interna no hay consenso. Como se comentó arriba, se han encontrado dos, tres y hasta cuatro factores en diferentes estudios (Sanz \& Vázquez, 1998). La estructura bifactorial coincide con los resultados hallados en otros estudios (Sanz \& Vázquez, 1998). Además, son susceptibles de recibir una etiqueta coherente con el constructo evaluado, ya que los dos factores resultantes parecen tener cierta lógica: el primero parece evaluar la presencia de creencias y sentimientos (desesperanza, culpabilidad, fracaso, etc.), y el segundo, la sintomatología vegetativa ( $\mathrm{p}$ ej; sueño, fatiga, ingesta, etc.).

Los valores hallados sobre el alfa ordinal y de alfa de Cronbach en el presente estudio resultaron algo superiores a los reportado en el estudio de Jurado et al (1998), y en las muestras españolas (Vázquez \& Sanz, 1997; Sanz \& Vázquez, 1998), pero se incluyen dentro del rango señalado por Beck, Steer y Garbin (1988). Además, debe destacarse los adecuados valores de confiabilidad observados en los factores.

Sobre la bondad de los items, puede ponerse en duda la pertinencia del reactivo 11 , primero, porque no pesa en ningún factor $\mathrm{y}$, segundo, porque al ser eliminado el valor del alfa de Cronbach de la escala total, subiría. Sin embargo, teniendo en cuenta que evalúa la presencia de irritabilidad -un sintoma muy relevante para detectar la presencia de depresión en adolescentes (Stringaris, Maughan, Copeland, Costello, \& Angold, 2013), y que habitualmente no se suele hacer uso de los factores del BDI- no consideramos prudente eliminar dicho item. Por otro lado, la ausencia de distribución normal de los reactivos es esperable debido a que aluden a la presencia de sintomas que no se espera que presenten la mayoría de los evaluados. Nótese que se hace también manifiesto en el valor de las medias (todas por debajo de uno), y las desviaciones estándar también indican poca variabilidad (todas por debajo de uno). 
En cuanto a los descriptivos, la ausencia de correlación entre la puntuación total y la edad no debe extrañar, puesto que la muestra era muy homogénea, todos eran jóvenes. Esta ausencia de relación entre edad y el BDI también es reportada en el estudio de Sanz y Vázquez (1998) realizado con estudiantes universitarios españoles. En el citado estudio tampoco se observaron diferencias en función del género, contrario a lo observado en el presente estudio, donde las mujeres obtuvieron mayores puntuaciones de sintomatología depresiva que los varones. Esto coincide con lo reportado por diferentes autores que señalan que las diferencias en la presencia de sintomatologia depresiva se inician en la adolescencia (Nolen-Hoeksema \& Girgus, 1994; Wade et al., 2002) y la prevalencia general de depresión es superior en mujeres respecto a los hombres a nivel mundial (Lim et al., 2018) y nacional (Bello et al., 2005).

Debe señalarse que la media general se halla justo en el limite entre depresión mínima y leve, tomando como referencia la clasificación de Beck y Steer (1993). Los resultados sobre la distribución por grupos derivados de la clasificación realizada por Beck y Steer (1993) son algo inferiores en gravedad respecto a lo reportado por Montoya et al., (2010), pero bastante más graves que los hallados por Sanz y Vázquez (1988). Los resultados del presente estudio pueden ser un indicador preocupante, y en especial si tenemos en cuenta que más de un $15 \%$ se sitúan en valores que indican la posible presencia de depresión moderada o grave. Además, haciendo uso del punto de corte de 18 sugerido por diversos autores (Chan, 1991; Kendall et al., 1987; Rudd \& Rajab, 1995), hasta un $13.3 \%$ muestran valores compatibles con probable cumplimiento de trastorno depresivo mayor según criterios DSM-5 (American Psychiatric Association, 2013). No obstante, debe recordarse que en México no se dispone de ningún estudio sobre validez diferencial para corroborar el 18 como punto de corte (o quizás otro) para detectar el posible trastorno depresivo mayor, tanto en población general como en estudiantes universitarios. Sería conveniente en un futuro realizar estudios sobre validez diferencial del BDI-I a través de entrevistas estructuradas, realizar el diagnóstico de trastorno depresivo mayor y compararlo con muestras con trastornos de ansiedad (sin comorbilidad de depresión) y con población sana (corroborado a partir de una evaluación exhaustiva).

Por otro lado, se hace necesario el estudio de la fiabilidad test-retest en esta población, así como realizar estudios sobre la sensibilidad al cambio de la escala en pacientes con trastornos depresivos que manifiestan mejoría clínicamente significativa después de recibir un tratamiento.

Debe mencionarse una notable limitación del presente trabajo, referida a que se utilizó solo estudiantes universitarios. Ello supone que la muestra presenta sesgos importantes, como el predominio de la juventud y del género femenino. Debido a ello, los resultados solo son representativos de población universitaria de Michoacán. Sería conveniente hacer uso de una población general con mayor variabilidad respecto a las características sociodemográficas para poder generalizar los resultados.

También es necesario comentar las limitaciones del BDI-I respecto al BDI-II, ya que en este último se pregunta por la presencia de exceso de alimentación y de sueño, y por alteraciones en el aparato motor. Aunque sean sintomas menos frecuentes, el BDI-I no es útil para detectar los sintomas atípicos de la depresión y las alteraciones motoras. 
Puede concluirse que la escala BDI, debido a los indicadores mostrados en la presente investigación, y tomando en consideración los que se han reportado en estudios previos, es un instrumento válido, fiable y de gran utilidad para evaluar la presencia de sintomatologia depresiva en población universitaria de Michoacán (México). Es importante señalar que la detección precoz de la sintomatología depresiva es fundamental por el elevado riesgo asociado al fracaso escolar, a otras patologías, e incluso al riesgo de suicidio. Asimismo, a partir de los resultados se infiere que un porcentaje importante de estudiantes universitarios pueden encontrarse en riesgo de padecer depresión, lo cual supone una señal de alerta que requiere urgente y eficaz atención.

\section{REFERENCIAS}

American Psychiatric Association (1995). Manual diagnóstico y estadístico de los trastornos mentales, cuarta edición (DSM-IV). Barcelona: Masson.

American Psychiatric Association. (2013). Diagnostic and statistical manual of mental disorders. Arlington, VA: American Psychiatric Publishing. https://doi.org/10.1176/appi.books.9780890425596

Beck, A. T., Rush, A. J., Shaw, B. F., \& Emery, G. (1979). Cognitive therapy of depression. Nueva York: Guilford.

Beck, A. T. \& Steer, R. A. (1993). Beck Depression Inventory. Manual. San Antonio, TX: The Psychological Corporation.

Beck, A. T., Steer, R. A., \& Brown, G. K. (1996). BDI-II. Beck Depression Inventory-Second Edition manual. San Antonio, TX: The Psychological Corporation. https://doi.org/10.1037/t00742-000

Beck, A. T., Steer, R. A., \& Brown, G. K. (2011). Manual. BDI-II. Inventario de Depresión de Beck-II (Adaptación española: Sanz, J., y Vázquez, C.). Madrid: Pearson.

Beck, A. T., Steer, R. A., \& Garbin, M. G. (1988). Psychometric properties of the Beck Depression Inventory: twenty-five years of evaluation. Clinical Psychology Review, 8, 77-100. https://doi.org/10.1016/02727358(88)90050-5

Beck, A. T., Ward, C. H., Mendelson, M., Mock, J., \& Erbaugh, J. (1961). An Inventory for Measuring Depression. Archives of General Psychiatry, 4(6), 561-571. https://doi.org/10.1001/archpsyc. 1961.01710120031004

Bello, M., Puentes, E., Medina-Mora, M. E., \& Lozano, R. (2005). Prevalencia y diagnóstico de depresión en población adulta en México. Salud Pública de México, 47(1), 4-11. https://doi.org/10.1590/S003636342005000100002

Bentler, P. M. (1990). Comparative fit indexes in structural models. Psychological Bulletin, 107, 238-246. https://doi.org/10.1037/0033-2909.107.2.238

Bollen, K. A. (1989). Structural equations with latent variables. Nueva York: Wiley. https://doi.org/10.1002/9781118619179

Botella, J. \& Ponte, G. (2011). Effects of the heterogeneity of the variances on reliability generalization: An example with the Beck Depression Inventory. Psicothema, 23(3), 516-522.

Chan, D. W. (1991). The Beck depression inventory: what difference does the Chinese version make? Psychological Assessment: A Journal of Consulting and Clinical Psychology, 3(4), 616-622. https://doi.org/10.1037/1040-3590.3.4.616

Dominguez-Lara, S. (2017). Fiabilidad y alfa ordinal. Actas Urológicas Españolas, 42(2), 140-141. https://doi.org/10.1016/j.acuro.2017.07.002

Estrada-Aranda, B. D., Delgado-Álvarez, C., Landero-Hernández, R., \& González-Ramírez, M. T. (2015). Propiedades psicométricas del modelo bifactorial del BDI-II (versión española) en muestras mexicanas de población general y estudiantes universitarios. Universitas Psychologica, 14(1), 125-136. https://doi.org/10.11144/Javeriana.upsy14-1.ppmb

Hamilton, M. (1960). A rating scale for depression. Journal of Neurology, Neurosurgery \& Psychiatry, 23(1), 56-62. https://doi.org/10.1136/jnnp.23.1.56

Hathaway, S. R. \& McKinley, J. (1943). Manual for administering and scoring the MMPI. Minneapolis: National Computer Systems.

Hu, L. \& Bentler, P. M. (1999). Cutoff criteria for fit indexes in covariance structure analysis: Conventional criteria versus new alternatives. Structural Equations Modeling, 6, 1-55. https://doi.org/10.1080/10705519909540118

IBM Corp Released, I. B. M. (2013). IBM SPSS statistics for Windows, version 22.0. Armonk, NY: IBM Corp.

Jurado, S., Villegas, M. E., Méndez, L. Rodríguez, F. Loperena, V., \& Varela, R. (1998). La estandarización del Inventario de Depresión de Beck para los residentes de la ciudad de México. Salud Mental, 21(3), 26-31.

Kang, H. J., Kim, S. Y., Bae, K. Y., Kim, S. W., Shin, I. S., Yoon, J. S., \& Kim, J. M. (2015). Comorbidity of depression with physical disorders: research and clinical implications. Chonnam medical journal, 51(1), 8-18. https://doi.org/10.4068/cmj.2015.51.1.8

Kendall, P. C., Hollon, S. D., Beck, A. T., Hammen, C. L., \& Ingram, R. E. (1987). Issues and recommendations regarding use of the Beck Depression Inventory. Cognitive therapy and research, 
11(3), 289-299. https://doi.org/10.1007/BF01186280

Kline, R. B. (1998). Principles and practice of structural equation modeling. Nueva York: Guilford.

Lara-Muñoz, M.C., Medina-Mora, M., Borges, G., \& Zambrano, J. (2007). El costo social de los trastornos mentales. Discapacidad y días productivos perdidos. Resultados de la Encuesta Nacional de Epidemiologia Psiquiátrica. Salud Mental, 30(5), 4-11.

Lim, G. Y., Tam, W. W., Lu, Y., Ho, C. S., Zhang, M. W., \& Ho, R. C. (2018). Prevalence of Depression in the Community from 30 Countries between 1994 and 2014. Scientific Reports, 8(1), 2861. https://doi.org/10.1038/s41598-018-21243-x

Mathers, C. D. \& Loncar, D. (2006). Projections of global mortality and burden of disease from 2002 to 2030. PLoS Medicine, e442. https://doi.org/10.1371/journal.pmed.0030442

Montgomery, S. A. \& Asberg, M. (1979). A new depression scale designed to be sensitive to change. The British Journal of Psychiatry, 134(4), 382-389. https://doi.org/10.1192/bjp.134.4.382

Montoya, L. M., Gutiêrrez, J. A., Toro, B. E., Briñón, M. A., Rosas, E., \& Salazar, L. E. (2010). Depresión en estudiantes universitarios y su asociación con el estrés académico. CES Medicina, 24(1), 7-17.

Muthén, L.K \& Muthén, B.O. (2011). Mplus user's guide (Version 7). Los Angeles, CA; Muthén \& Muthén.

Newby, J. M., McKinnon, A., Kuyken, W., Gilbody, S., \& Dalgleish, T. (2015). Systematic review and metaanalysis of transdiagnostic psychological treatments for anxiety and depressive disorders in adulthood. Clinical psychology review, 40, 91-110. https://doi.org/10.1016/j.cpr.2015.06.002

Nolen-Hoeksema, S. \& Girgus, J. S. (1994). The emergence of gender differences in depression during adolescence. Psychological Bulletin, 115(3), 424-443. https://doi.org/10.1037/0033-2909.115.3.424

Organización Mundial de la Salud. (2015). La depresión. Recuperado de http://www.who.int/mediacentre/factsheets/fs369/es/

Radloff, L. S. (1977). The CES-D scale: A self-report depression scale for research in the general population. Applied psychological measurement, 1(3), 385-401. https://doi.org/10.1177/014662167700100306

Rudd, M. D. \& Rajab, M. H. (1995). Specificity of the Beck Depression Inventory and the confounding role of comorbid disorders in a clinical sample. Cognitive Therapy and Research, 19(1), 51-68. https://doi.org/10.1007/BF02229676

Sanz, J. (2013). 50 años de los Inventarios de Depresión de Beck: consejos para la utilización de la adaptación española del BDI-II en la práctica clínica. Papeles del psicólogo, 34(3), 161-168.

Sanz, J. \& Garcia-Vera, M. P. (2013). Rendimiento diagnóstico y estructura factorial del Inventario para la Depresión de Beck-Segunda Edición (BDI-II) en pacientes españoles con trastornos psicológicos. Anales de Psicologia, 29(1), 66-75. https://doi.org/10.6018/analesps.29.1.130532

Sanz, J., \& Vázquez, C. (1998). Fiabilidad, validez y datos normativos del inventario para la depresión de Beck. Psicothema, 10(2), 303-318.

Stringaris, A., Maughan, B., Copeland, W. S., Costello, E. J., \& Angold, A. (2013). Irritable mood as a symptom of depression in youth: prevalence, developmental, and clinical correlates in the Great Smoky Mountains Study. Journal of the American Academy of Child \& Adolescent Psychiatry, 52(8), 831-840. https://doi.org/10.1016/j.jaac.2013.05.017

Vázquez, C., \& Sanz, J. (1997). Fiabilidad y valores normativos de la versión española del inventario para la depresión de Beck de 1978. Clínica y Salud, 8(3), 403-422. https://doi.org/10.1097/00004583200202000-00013

Wade, T. J., Cairney, J., \& Pevalin, D. J. (2002). Emergence of gender differences in depression during adolescence: National panel results from three countries. Journal of the American Academy of Child \& Adolescent Psychiatry, 41(2), 190-198

Zung, W. W. K. (1965). A Self-Rating Depression Scale. Archives of General Psychiatry, 12(1), 63-70. https://doi.org/10.1001/archpsyc. 1965.01720310065008

Recibido 20-03-2020| Aceptado 24-07-2020 